\title{
Évolution des groupes axiaux pour l'équipement des installations marémotrices
}

\author{
Development of axial flow machines for use in tidal power plants
}

\author{
J. Bosc \\ Directeur technique \\ Neyrpic
}

\author{
L. Megnint \\ Chef de Département D.C.H. \\ Neyrpic
}

Parmi les formes d'énergie nouvelles (solaire, éolienne, géothermique), l'énergie marémotrice occupe une place de choix car elle profite de tous les développements antérieurs de l'énergie hydraulique et de ses qualités reconnues : gratuité du combustible et facilité d'adaptation aux fluctuations de la demande.

Les sites marémoteurs à travers le monde sont au nombre d'une douzaine et constituent un potentiel de plusieurs centaines de TWh par an.

Les problèmes fondamentaux qui se posaient il $\mathrm{y}$ a plusieurs décennies lors des études de la première installation marémotrice se trouvent aujourd hui résolus, comme le confirme l'exploitation des turbines de La Rance pendant plus de quinze ans. Mais si ce succès permet d'asseoir les réalisations futures sur des bases sûres, la recherche de l'économie optimale reste pour les usines marémotrices comme pour les installations de rivière le principal souci des maitres d'œuvre et des constructeurs.

Les prochaines usines marémotrices seront équipées avec des groupes axiaux qui se révèlent les mieux adaptés à ce type d'installations de basses chutes.

Le développement des turbomachines axiales s'est fait selon deux voies principales:

- les groupes bulbes:

- les groupes avec alternateur périphérique.

Jusqu’à présent, les efforts se sont surtout portés sur les groupes bulbes, et les progrès réalisés en puissance et dimensions permettent d'envisager avec succès l'équipement d'installations marémotrices puissantes avec ce type de machines. Dans l'état actuel de la technique, on peut projeter des groupes munis de roues atteignant 8 à $9 \mathrm{~m}$ de diamètre.

Selon les fonctionnements envisagés pour les installations maremotrices, trois types de turbines peuvent être utilisés :

- distributeur et pales réglables;

- distributeur fixe et pales réglables;

- distributeur mobile et pales fixes.
Les deux premiers types conviennent pour des fonctionnements en simple et en double effet; le dernier ne marche qu'en turbine simple effet.

Le développement des groupes avec alternateur périphérique situé autour de la roue de la turbine ne s'est pas poursuivi de façon continue comme celui des bulbes.

La complexité de telles machines a conduit à limiter le diamètre des roues comportant des pales réglables : à notre connaissance, à l'heure actuelle le plus grand diamètre d'une turbine en fonctionnement atteint $3,6 \mathrm{~m}$. Une installation est prévue avec une roue à pales fixes de 7,6 $\mathrm{m}$ de diamètre; mais elle n'est pas encore en service.*

Il faudra attendre de nombreuses années d'exploitation de ce type de machines de grands diamètres avant que les maîtres d'œuvre n'envisagent leur utilisation pour l'équipement d'usines marémotrices puissantes, qui exigent des investissements énormes.

$\mathrm{Si}$ le succès de La Rance et le développement des groupes bulbes ont ouvert indiscutablement des perspectives certaines, il n'est jamais venu à l'esprit des constructeurs qui participèrent à ces actions de prétendre qu'il suffirait de copier La Rance pour assurer le développement des centrales marémotrices.

Parmi les paramètres caractéristiques de l'équipement, deux retiennent particulièrement l'attention :

- le choix du diamètre de la roue est très important puisqu'il fixe l'échelle du génie civil de l'usine. La tendance vers les grands diamètres semble être une nécessité économique;

- la chute nominale, égale à la chute minimale nécessaire pour obtenir la puissance nominale, doit être fixée assez basse pour bien passer les petites marées mais suffisante pour ne pas écrêter les fortes.

Ces deux paramètres conditionnent la vitesse de rotation du groupe et partant, les dimensions de l'alternateur.

Comme les sites marémoteurs sont caractérisés par des

\footnotetext{
* Cette installation est maintenant en fonctionnement.
} 
chutes variant entre zéro et un maximum de 13 à $14 \mathrm{~m}$, les fonctionnements à faible chute influencent fortement la productivité des installations marémotrices : les solutions retenues pour le matériel électromécanique doivent donc être bien adaptées à ces conditions.
Deux dispositions sont possibles : - utilisation de multiplicateurs de vitesse; - fonctionnement des groupes à vitesse variable en utilisant des convertisseurs statiques de fréquence, à puissance totale ou à puissance partielle.

\section{Multiplicateurs de vitesse}

Ils apportent une réponse technologique à la nécessité d'améliorer l'économie d'ensemble des projets d'usines marémotrices.

A titre d'exemple, l'un des projets d'aménagement de la centrale marémotrice de Garolim en Corée, établi en 1981, comporte 24 groupes d'une puissance de $20 \mathrm{MW}$. Les turbines, d'un diamètre de $8 \mathrm{~m}$, tourneraient à $50 \mathrm{tr} / \mathrm{min}$ en entraînant un alternateur à $300 \mathrm{tr} / \mathrm{min}$, au moyen d'un multiplicateur de rapport 6 .

(L'évolution des études depuis 1981 permet d'envisager à l'heure actuelle des alternateurs tournant à $600 \mathrm{tr} / \mathrm{min}$ avec un multiplicateur à deux étages).

Les machines de puissance identique munies d'alternateur à attaque directe tourneraient sensiblement à la même vitesse et comporteraient un stator ayant la forme d'un disque plat de grand diamètre (de l'ordre de $10 \mathrm{~m}$ ) et de faible longueur. Le diamètre important de l'alternateur risque d'entraîner un élargissement du pertuis d'entrée du groupe avec pour conséquence un accroissement de l'entr'axe des machines.

De plus, les conditions de réalisation de l'alternateur :

- faible pas polaire surtout avec des réseaux à $60 \mathrm{~Hz}$,

- conditions de transport imposant l'exécution de la carcasse en plusieurs éléments assemblés sur site,

- réfrigération malaisée,

conduisent à une technologie mal adaptée et à un prix trop élevé.

L'emploi d'un multiplicateur permet l'utilisation d'un alternateur plus classique, de meilleur rendement et d'un prix plus faible.

Il existe une valeur de la vitesse de rotation pour laquelle les prix :

- d'une part de la solution alternateur à attaque directe, - d'autre part de la solution alternateur plus multiplicateur,

sont sensiblement les mêmes. Cette limite semble être actuellement fixée aux alentours de $80 \mathrm{tr} / \mathrm{min}$.

$\mathrm{Si}$ le multiplicateur introduit une perte supplémentaire (rendement $99,3 \%$ pour un multiplicateur à 1 étage, 98,7\% pour un multiplicateur à 2 étages), celle-ci est largement compensée par le gain sur l'alternateur dû à sa vitesse plus élevée.

En conclusion, l'emploi d'un groupe bulbe avec multiplicateur de vitesse permet de rentabiliser les aménagements sous faible chute, ce qui présente un intérêt certain pour les marémotrices.

Pour bien mettre en évidence toutes les implications technologiques liées à cette conception, nous nous sommes fixé pour objectif l'étude de l'implantation d'un multiplicateur dans une machine existante; et bien que la vitesse de $93,75 \mathrm{tr} / \mathrm{min}$ soit au-dessus de la limite économique de $80 \mathrm{tr} / \mathrm{min}$ citée précédemment, nous avons retenu pour cadre un groupe marémoteur de La Rance.

Cette étude, matérialisée par la figure 1 met bien en évidence la comparaison des deux solutions :

- alternateur à attaque directe $\quad n=93,75 \mathrm{tr} / \mathrm{min}$, - alternateur rapide avec multiplicateur

$n=92,25 / 500 \mathrm{tr} / \mathrm{min}$.

Ses conclusions se résument de la façon suivante :

- la puissance est conservée

- le nouveau bulbe amont, plus encombrant en longueur, s'accomode de la place impartie, notamment vis-à-vis du batardeau côté bassin

- le conduit hydraulique est peu affecté. Et même, si l'étude plus poussée de l'alternateur $500 \mathrm{tr} / \mathrm{min}$ permet d'accepter un diamètre d'enceinte égal au diamètre de carcasse actuel, les sections de passage d'eau seront absolument inchangées.

- la plupart des organes mécaniques nobles sont conservés, en particulier l'arbre turbine

- la régulation est peu affectée

- on peut utiliser pour le multiplicateur la même huile que celle des paliers

- le processus et l'outillage de montage sont inchangés

- le génie civil n'est pas affecté

- les nouveaux organes alternateur et multiplicateur sont classiques

- l'accès à tous les organes situés à l'intérieur du bulbe est considérablement amélioré. Il est même possible en marche car il n'y a pas d'alternateur à traverser. Il n'y a plus d'air surpressé.

Les approches économique et technologique ont mis en évidence l'intérêt et la faisabilité des groupes bulbes avec multiplicateur. Encore faut-il s'assurer que cette disposition soit fiable et que le multiplicateur n'introduise pas un risque de mauvais comportement en service.

Les constructeurs de multiplicateurs ont fait de grands progrès ces dernières années et les conceptions qu'ils ont retenues permettent d'envisager raisonnablement des puissances atteignant $40 \mathrm{MW}$.

Les références actuelles sont à hauteur de :

$25 \mathrm{MW} 60 / 906 \mathrm{tr} / \mathrm{min}$ pour la propulsion navale, $14 \mathrm{MW} 68 / 750 \mathrm{tr} / \mathrm{min}$ pour les turbines hydrauliques.

Le type retenu est le multiplicateur à planétaire : le nombre de satellites peut varier de 3 à 9 . 
Les progrès ont été réalisés dans deux domaines principaux :

a) Liberté d'adaptation des matériels aux types de sollicitations

Que ce soit dans la marine ou dans l'hydraulique, on a des vitesses lentes, des flexions d'arbre importantes (de l'ordre du millimètre), des gros diamètres avec jeux aux paliers relativement grands.

Par ailleurs, les transitoires hydrauliques sont de l'ordre de la seconde et les transitoires thermiques de l'ordre de la demi-heure.

Le multiplicateur doit pouvoir s'adapter à toutes ces sollicitations. Pour ce faire, tout le multiplicateur est suspendu sur l'arbre turbine, qui supporte donc le porte-satellites, le pignon solaire et les couronnes fixes.

Deux adaptations sont prévues :

- côté grande vitesse, par un arbre flottant assurant la liaison entre le pignon solaire et l'arbre alternateur et réalisant un accouplement à fonction cardan, très rigide en torsion et souple en flexion

- au niveau des pièces fixes, le carter est libre en flexion, mais sa rotation est empêchée par un système de bielles tangentielles ou de lames flexibles. b) Réalisation des parties actives

Tous les gains vont dans le sens d'une meilleure tenue en service des dentures :

- amélioration des traitements thermiques de façon à obtenir une matière homogène dans la masse

- taillage avec correction de denture pour tenir compte de la flexion de la dent en service

- précision de réalisation et finition des surfaces actives.

A ces améliorations de réalisation, il convient d'ajouter toutes les recherches faites sur la qualité d'huile nécessaire pour assurer une bonne lubrification des surfaces en contact malgré des pressions d'appui importantes.

Pour être totalement complet sur les groupes avec multiplicateur, précisons que pour éviter de soumettre l'alternateur aux conditions sévères de fonctionnement à l'emballement (ce qui impose de le dimensionner pour ce cas), un dispositif de débrayage est en cours d'étude. Ce mécanisme disposé sur l'arbre grande vitesse, du type débrayage en marche et réarmement à l'arrêt, est commandé par un détecteur de survitesse. Il est impératif que ce dispositif soit d'une haute fiabilité.

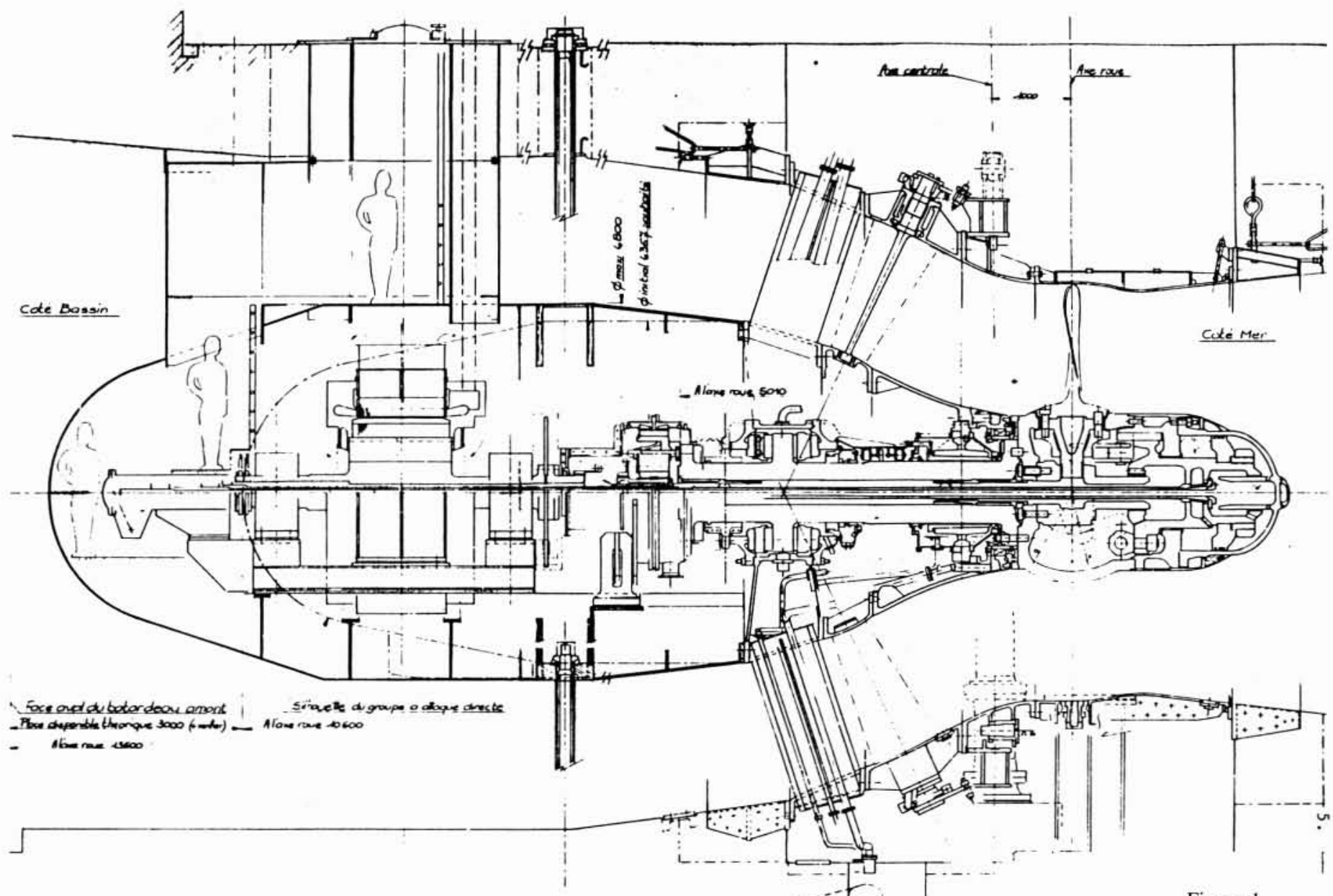

Figure 1 


\section{Emploi de la vitesse variable sur les groupes marémoteurs}

Le fonctionnement en turbine des groupes marémoteurs s'effectue dans une très large gamme de chutes pouvant aller du voisinage de la chute maximum à la chute minimum turbinable, c'est-à-dire celle pour laquelle l'emballement maximum maximorum correspond à la vitesse de synchronisme.

Le fonctionnement à haute chute s'effectue généralement dans la zone des bons rendements alors que les basses chutes se font avec des rendements décroissant avec la chute.

Le ralentissement de la vitesse du groupe au fur et à mesure que la chute diminue permet d'augmenter le rendement des machines et la production du cycle.

Le même ralentissement peut être bénéfique non seulement dans le fonctionnement en pompe normale, mais aussi en pompe accélératrice, c'est-à-dire lorsque la charge à l'aspiration est supérieure à la charge au refoulement.

Différents moyens peuvent être envisagés pour obtenir des groupes fonctionnant à vitesse variable : génératrices à courant continu, alternateurs à plusieurs vitesses, convertisseur statique de fréquence, etc.

Une solution très voisine consiste à redresser le courant alternatif produit par les groupes, à transporter à longue distance ce courant continu et à le transformer en courant alternatif au voisinage des lieux de consommation. $\mathrm{Ce}$ système fonctionne depuis plusieurs années entre réseaux, ou entre centrales et réseaux, à l'échelle de plusieurs TW.

Pour les groupes marémoteurs où les puissances à transmettre sont de l'ordre de quelques dizaines de MW, la solution qui semble la plus intéressante est celle d'un alternateur accouplé à un convertisseur statique de fréquence (C.S.F.).

\section{Le convertisseur statique de fréquence :}

Le C.S.F.est en gros ủn redresseur qui transforme en courant continu le courant alternatif produit par l'alternateur.

Ce courant continu est ensuite transformé en courant alternatif par un onduleur à la fréquence du réseau.

Le système peut être réversible, ce qui permet de l'utiliser dans le fonctionnement en pompe.

Les C.S.F. ont fait leur peuve avec des puissances de quelques MW pour l'alimentation des moteurs de laminoir à vitesse variable, puis comme lanceurs en pompe de groupes turbines-pompes. Dans ce cas, la puissance atteinte est d'une vingtaine de MW (centrales de Montézic et du Cheylas).

Les matériels sont encore assez volumineux, mais dans le cas d'une centrale marémotrice, rien ne s'oppose à leur installation à terre, près du poste.

\section{a) Utilisation}

Pour simplifier, étudions le cas d'un groupe à distributeur fixe/pales mobiles.

Généralement, dans le cas d'un fonctionnement en turbine directe ou turbine inversée, les points de fonctionnement sur un diagramme en coordonnées réduites

$$
\text { (abscisse } n_{11}=\frac{n D}{\sqrt{H}} \text { et ordonnée } Q_{11}=\frac{Q}{D^{2} \sqrt{H}} \text { ) }
$$

se placent dans un triangle curviligne

$A B C$ (voir figure 2).

$A B$ est la ligne de rendement maxi pour $n_{11}$ donné

$B C$ est la ligne de saturation de puissance à $n_{11}$ donné

$C A$ est la ligne de limitation de puissance de l'alternateur à vitesse constante.

Considérons un point de fonctionnement située en $I$ d'abscisse $n_{1}$.

Un ralentissement de la vitesse de fonctionnement entraînera le passage de l'abscisse $n_{1}$ à $n^{\prime}{ }_{1}$.

Si la position des pales ne bouge pas, on va se déplacer de $I$ en $I_{1}$ sur une équipale $i_{1}$. On pourra obtenir une augmentation du rendement, mais elle sera accompagnée d'une réduction du débit et il pourra en résulter une diminution de puissance.

Par contre, en ouvrant les pales, on pourra se placer en un point du segment $I_{1} I_{2}$, ce qui permet de fonctionner au même débit qu'en $I$ ou même à un débit supérieur. En particulier, les points entre $I_{3}$ (maxi de rendement) et $I_{2}$ sont très intéressants. Compte tenu de la puissance maxi de l'alternateur à cette vitesse, le gain de rendement sera d'autant plus élevé que le point de fonctionnement se rapprochera de la courbe $\mathrm{EF}$, lieu des points donnant le meilleur rendement à débit donné. Ce type de fonctionnement peut d'ailleurs amener à prévoir une surouverture des pales qui serait utilisée lors du fonctionnement à vitesse réduite, donc sous chute réduite, lorsque les problèmes de cavitation sont moins à craindre.

Un raisonnement analogue peut s'appliquer aux fonctionnements en pompe, mais le fonctionnement sous hauteur de refoulement très faible augmente sans doute l'intérêt d'une surouverture des pales.

\section{b) Puissance du convertisseur}

Dans le cas de machine à vitesse fixe, pour des raisons économiques on ne dimensionne généralement pas les alternateurs pour la puissance maximum que pourrait développer la turbine sous la chute maximum. Le temps d'utilisation de cette puissance serait faible, mais il faudrait un calage très profond de l'usine pour mettre les groupes à l'abri de la cavitation pendant ce fonctionnement. Donc, au-delà d'une chute dite nominale, la puissance de l'alternateur est limitée à la valeur maximum atteinte sous cette chute nominale (point $C$ du diagramme à vitesse fixe). Lorsque la chute augmente, le point caractéristique passe de $C$ en $A$.

Au voisinage de ces chutes (courbe $A C$ ), les rendements de la turbine sont proches du maximum. Si l'on introduit un convertisseur de fréquence, le gain de rendement de la turbine sera faible et peut-être même effacé par la perte de rendement induite par le C.S.F. 
Si l'on ajoute que l'investissement représenté par le C.S.F. est loin d'être négligeable, il nous semble qu'il est sans doute intéressant d'utiliser le dispositif décrit ciaprès. Lors du fonctionnement aux fortes chutes et fortes charges, les groupes équipés d'alternateurs triphasés débitent directement sur le réseau (à $50 \mathrm{~Hz}$ par exemple). $\mathrm{Ce}$ n'est que lorsque la chute diminue, entraînant une réduction de la puissance, qu'il peut devenir intéressant d'utiliser un convertisseur de fréquence; par exemple à la moitié ou au 1/4 de la charge nominale.

Une étude économique devrait pouvoir déterminer la puissance maximum à installer en convertisseur.

$\mathrm{Ce}$ fonctionnement avec C.S.F. à puissance réduite nous parait favoriser les solutions avec turbines à distribu- teur mobile et pales réglables et à distributeur fixe et pales réglables. Par contre, la solution distributeur mobile et pales fixes exigerait plutôt un surdimensionnement de l'alternateur et du C.S.F. éventuel.

La réduction de puissance du C.S.F. ne devrait pas affecter le fonctionnement en pompe qui s'effectue à puissance réduite. De plus, le C.S.F. résoud élégamment le problème du démarrage en pompe des groupes marémoteurs, tout au moins dans le cas des pales mobiles.

En conclusion, il serait intéressant d'entreprendre une étude des cycles marémoteurs avec C.S.F. Le gain de production à en retirer sera surtout intéressant pour les marées de faible amplitude, et donc de faible production.

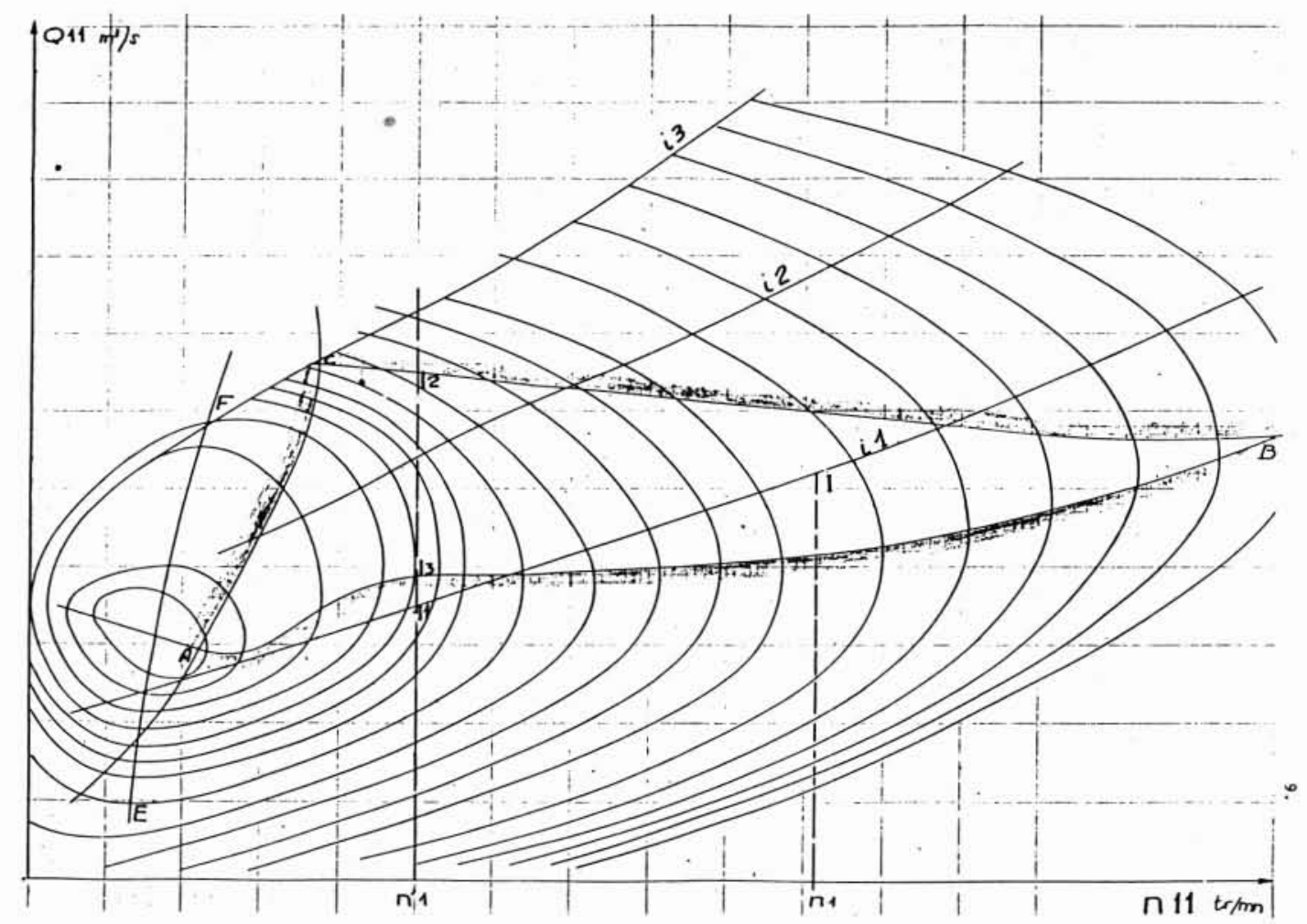

Figure 2

\section{Discussion}

\section{Président : M.R. VULSER}

L'exposé a été lui aussi présenté à plusieurs voix par M. Bosc, Directeur technique de Neyrpic, qui a montré l'intérêt des multiplicateurs de vitesse et décrit un projet de ce type, M. MegniNT, Chef de Département à Neyrpic, qui a expliqué le fonctionnement à vitesse variable par l'emploi de convertisseurs statiques de fréquence (C.S.F.).
La discussion comporte essentiellement une question de $\mathrm{M}$. BOURDON sur les limites des appareils en fonctionnement : $28 \mathrm{MW}$ pour les C.S.F. (démarreurs de Montézic et du Cheylas) - $15 \mathrm{MW}$ pour les multiplicateurs. 


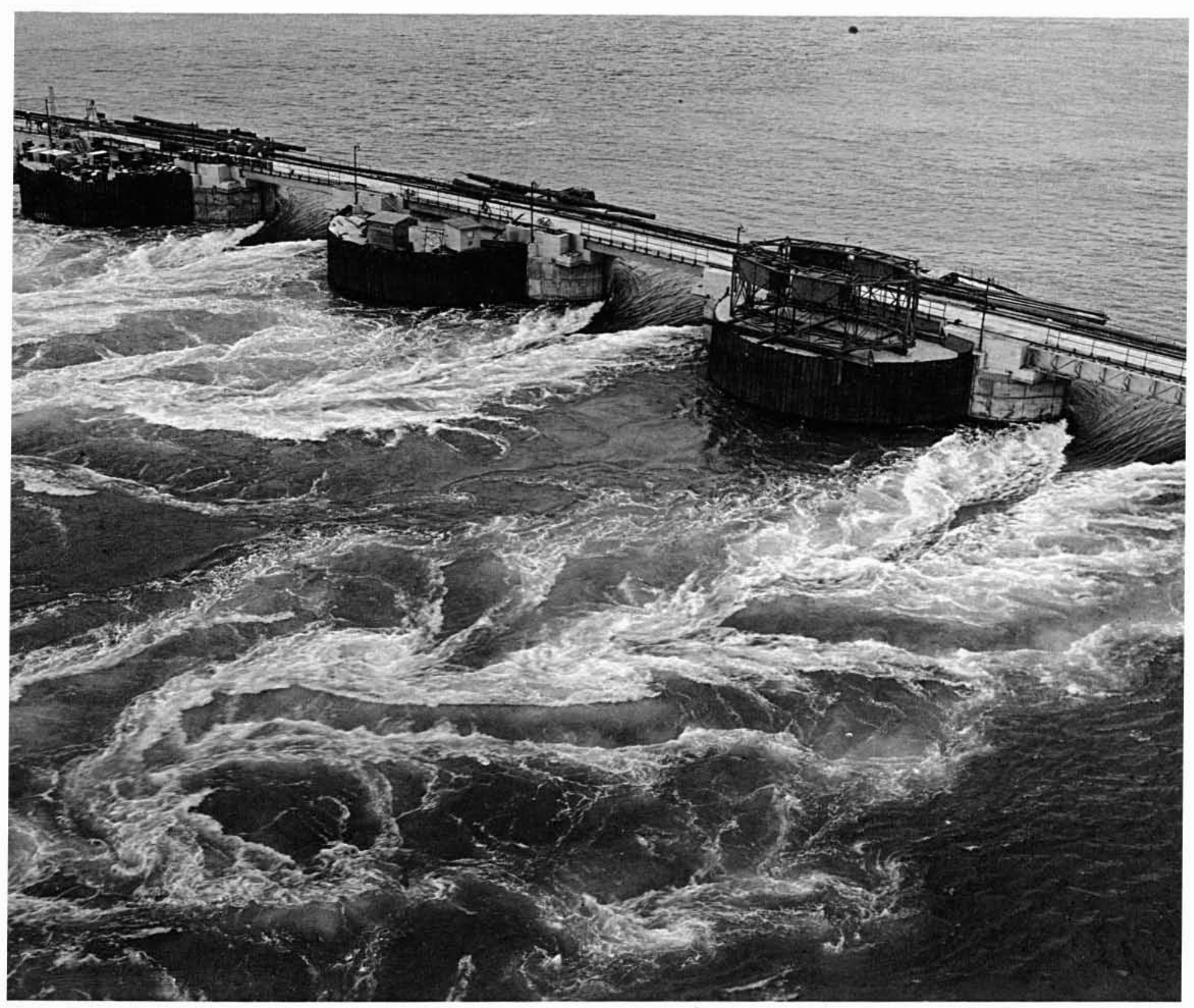

